www.nature.com/jes

\title{
EDITORIAL
}

\section{Scientific gains in exposure science}

Journal of Exposure Science and Environmental Epidemiology (2008) 18, 1; doi:10.1038/sj.jes.7500642

Last year was a tremendous year in the field of exposure science and for the Journal of Exposure Science and Environmental Epidemiology (JESEE). We published articles on a wide range of important and cutting-edge subjects, including EMF, environmental chemical, criteria pollutant and particulate matter exposures; novel modeling techniques and investigations of the roles of GIS, multimedia assessment techniques, and dietary and behavioral patterns in exposure science. We were fortunate to have three additional issues this year, one special issue on biomonitoring, a supplement on the REACH legislation in Europe and another on ambient air. Overall, the collection of papers published in JESEE was high quality and scientifically sound, yet remained on the forefront of interest in exposure science, some even evoking interesting scientific exchange and debate. Clearly, the process of scientific progress and peer review is working well in JESEE.

Because of the hard work of our editorial team including that of our Editor Emeritus, Edo Pellizari, authors, reviewers, and readers, our 2006 impact factor increased to 2.4. In addition, our ISI rankings in the three major areas surrounding exposure science - environmental sciences, toxicology, and public/environmental/occupational health - increased dramatically, putting us in the top $30 \%$ in all areas. As a result of the hard work of many individuals, we are receiving more high-quality papers for potential publication and are asking more of our peer reviewers and readership. Because the peer-review process is the fundamental underpinning of science, we ask you to consider reviewing articles in JESEE this year, if asked. Thoughtful and critical reviews are necessary to ensure that only the soundest science is published. As scientists, it is not only our privilege to review our colleagues' manuscripts, but also our duty to ensure the scientific process is complete and beyond reproach. Thank you to all of those who have served as peer reviewers. We hope you continue to serve in such an important role and encourage others to do so as well.

Another important occurrence in the journal this year is the expansion of our editorial board and the institution of a rotating system of board members. We are thankful for the dedicated service of Lisa Melnyk, George Thurston, and Debbie Bennett who retired from the editorial board this year. We also welcome six new members: John Adgate, Tina Bahadori, Sean Hays, Holger Koch, Marcia Nishioka, and Yuri Bruin de Bruinen. We believe that the expansion of our board and the rotation of members will bring more rapid decisions and will help ensure that only the top articles are published in JESEE.

We look forward to a fruitful year in the field of exposure science in 2008. We encourage the submission of articles in cutting-edge areas of exposure science and hope to provide our readership with even more quality scientific publications to guide their research.

Happy New Year!
Dana B. Barr PhD Editor-in-Chief 